\title{
Impulse Noise Removal Based on Hybrid Genetic Algorithm
}

\author{
Nail Alaoui ${ }^{1 *}$, Arwa Mashat ${ }^{2}$, Amel Baha Houda Adamou-Mitiche ${ }^{1}$, Lahcène Mitiche ${ }^{1}$, Aicha Djalab $^{3}$, Sara Daoudi $^{4}$, \\ Lakhdar Bouhamla ${ }^{1}$ \\ ${ }^{1}$ Laboratoire de Recherche Modélisation, Simulation et Optimisation des Systèmes Complexes Réels, Université ZIANE \\ Achour de Djelfa, Ain Chih, Djelfa 17000, Algeria \\ ${ }^{2}$ Faculty of Computing \& Information Technology, King Abdulaziz University, P.O. Box 344, Rabigh 21911, Saudi Arabia \\ ${ }^{3}$ Department of Electrical Engineering, Faculty of Technology, University of Djelfa, P. O. B. 3117, Djelfa, Algeria \\ ${ }^{4}$ RCAM Laboratory Dept of Electronics, Djillali Liabès University Sidi Bel Abbes, Sidi Bel Abbes 22000, Algeria
}

Corresponding Author Email: n.alaoui@univ-djelfa.dz

https://doi.org/10.18280/ts.380436

Received: 21 November 2020

Accepted: 12 July 2021

\section{Keywords:}

image denosing, noise removal, impulse noise, salt and pepper noise, genetic algorithm

\begin{abstract}
In this paper, we introduce a new method, impulse noise removal based on hybrid genetic algorithm (INRHGA) to remove impulse noise at different noise densities of noise while preserving the main features of the image. The proposed approach merges the genetic algorithm and methods for filtering images that are combined into the population as essential solutions to create a developed and improved population. A set of individuals is developed into a number of iterations using factors of crossover and mutation. Our method develops a group of images instead of a set of parameters from the filters. We then introduced some of the concepts and steps of it. The proposed algorithm is compared with some image denoising algorithm. By using Peak Signal to Noise Ratio (PSNR), structural similarity (SSIM). For example, for Lenna image with $60 \%$ salt and pepper noise density, PSNR, SSIM results of AMF, MDBUTMFG and NAFSM methods are 20,39/ 28.74/ 29.85 and 0.5679/ 0.8312/ 0.8818 respectively, while PSNR, SSIM results of the proposed algorithm are 29.92 and 0.8838 , respectively. Experimental results indicate that INRHGA is very effective and visually comparable with the above-mentioned methods at different levels of noise.
\end{abstract}

\section{INTRODUCTION}

Impulse noise removal is one of the essential issues in image processing, many approaches have been proposed to suppression of noise in digital images from the literature, and however, eliminate noise from digital images is still a difficult problem [1-7].

Different sensors, for example, laser scanners, medical scanners, cameras, and weather satellites, can obtain digital images, but these images might inherently be polluted by noise during acquisition compression processes, transmission [8-11]. It is essential to remove the noise while retaining the basic features of the image, such as edges and corners.

Some nonlinear filters have been suggested for the recuperation of images corrupted by impulse noise. The average filter, as well as its derivatives, are most common in image filtering.

The Median Filter (MF) method utilizes a fixed Window Size and is used to all pixels [12].

There are many common noise filters. For instance, the Adaptive Media Filter (AMF), which uses an adaptive window size, unlike MF which uses a fixed window size. However, although AMF is very effective at removing high-density Impulse noise in images compared to MF, but if the window size is large, it prevents us from finding pixels that match the pixels of the original image [13].

In high-density Impulse noise, a Modified Decision-Based Unsymmetrical Trimmed Median Filter (MDBUTMF) is used, where an adaptive window is used to identify and remove noisy pixels. Then, the MF is utilized to them [14]. Whereas Noise adaptive fuzzy switching median filter (NAFSM) applies the histogram to detect noise pixels in the noisy image, then these pixels are changed by applying MF or estimated according to their neighbors' values [15].

Other methods study the problem of removing noise from images as an optimization problem. Hence, genetic methods have been successfully applied. Some of the most modern papers involving genetic algorithms are [16-25].

Despite this interest, do not exist GAs intended to remove impulse noise in gray images by evolving images.

In this work, we describe a new genetic algorithm called INRHGA that removes impulse noise in gray images.

Our work is inspired by the approaches [19-21], but we address the problem from a different perspective. The fundamental idea is to merge the output images of two of the best methods found in the literature into the initial population of the Genetic Algorithm as essential solutions. Evolution happens for a certain number of epochs aiming to find the best image. During this process, a specific crossover and mutation are applied. Our method develops a group of images instead than a group of parameters from the filters. Our experimental results show that the proposed algorithm improves, in general, the performance for both image denoising and preservation of images details.

\section{METHODOLOGIES}

This section describes our proposed genetic algorithm that 
suppresses noise in an image. The input of this proposed method is an image gray-scale $\mathrm{N}(\mathrm{x}, \mathrm{y})$ perturbed through impulse noise. Enhanced image of $\mathrm{N}(\mathrm{x}, \mathrm{y})$ is the output each individual in the proposed algorithm of the initial population is represented a denoised image of $\mathrm{N}(\mathrm{x}, \mathrm{y})$.

In this paper, we employ the following two models for impulse noise in gray images:

i. $\quad$ Noise Model 1: Salt-and-pepper Impulse noise. For this model, the image is corrupted value by noise can only be 0 or 255 with the same probability.

The probability distribution function is given by:

$$
f(x)=\left\{\begin{aligned}
\frac{p}{2} \text { for } N & =0 \\
1-p \text { for } N & =O(i, j) \\
\frac{p}{2} \text { for } N & =255
\end{aligned}\right.
$$

where, $p$ is the noise density in the image.

For each original image pixel at location $(i ; j)$ the intensity value is $O(i, j)$, the corresponding pixel of the noisy image is given by $N(i, j)$.

ii. Noise Model 2: For this model, Here corrupted image have fixed value for salt (i.e. 255) and pepper (i.e. 0) noise with unequal probability.

The probability distribution function is given by:

$$
f(x)=\left\{\begin{array}{c}
P_{1} \text { for } N=0 \\
1-p \text { for } N=O(i, j) \\
P_{1} \text { for } N=255
\end{array}\right.
$$

where, $p=p 1+p 2$ is the noise density in the image and $p 1 \pm$ p2.

Hybrid genetic algorithm is guided by the objective function expressed in Eq. (3).

$$
\begin{gathered}
\text { ObjectFitness }(F)=\lambda|I-N|+ \\
\left(\sum_{\Omega} \sqrt{1+\beta^{2}|\nabla I|^{2}}\right)
\end{gathered}
$$

which is an edge aware feature preserving diffusion flow function stems from the studies [26, 27]. The term $I(x, y)$ is the image being recovered, $\mathrm{N}(\mathrm{x}, \mathrm{y})$ the noisy image, $\beta$ and $\lambda$ are balancing parameters and $\Omega$ is the set of all points in the image. where, $\lambda>0$ and $1 \leq \beta \leq 2$ from [26].

By minimizing Eq. (1), we are basically trying to reduce the total variation of the image while preserving fidelity in relation to the original image.

The general execution for the proposed algorithm consists of the following steps:

\section{INRHGA Algorithm Steps}

Step 1. (Input image) Read a noisy image $\mathrm{N}(\mathrm{i}, \mathrm{j})$ is represented by an array of pixels $\mathrm{N}(\mathrm{i}, \mathrm{j})$ where $\mathrm{i}$ and $\mathrm{j}$ range from 0 to 255 and 255, respectively.

Step 2. (Initialization) Execute filters MDBUTMFG and NAFSM over noisy image $\mathrm{N}(\mathrm{i}, \mathrm{j})$ to create two new images. $\mathrm{N}_{\text {MDBUTMFG }}$ and $\mathrm{N}_{\mathrm{NAFSM}}$, respectively.

The first two individuals (images) of the population, denoted as $\mathrm{N}_{\text {MDBUTMFG }}, \mathrm{N}_{\mathrm{NAFSM}}$, are the resulting images after applying the following filters: MDBUTMFG and NAFSM to noisy images (Input image)

Then, execute a pixel recombination procedure that randomly exchanges pixels between $\mathrm{N}_{\text {MDBUTMFG }}$ and $\mathrm{N}_{\mathrm{NAFSM}}$ to create an initial population of size Ps.
Step 3. (Evaluation) Use Eq. (1) to evaluate the fitness of the initial population.

Step 4. (Selection) Select a pair of the initial population by a Roulette Wheel selection.

Step 5. (Crossover) crossing pairs of selected parents to create offspring. We have applied the same crossover factors proposed by Ahmed and Das [4].

Step 6. (Mutation) Mutate each offspring with probability $P_{m}$ through the execution of one of the filters MDBUTMFG or NAFSM selected randomly.

Step 7. (Update population) Choose the best Ps individuals of previous generation and their offspring according to their fitness, then retain these individuals for the next iteration as an initial population.

Step 8. Steps 3 to 7 are repeated until iter $_{\max }$ is reached.

The best individual according to the fitness value in the last generation is considered as the denoised image $I(i, j)$.

This configuration was based on some empirical tests that took into account the computational time spent by executing the INRHGA combined with the other denoising methods. For example, it is not possible to set a large-sized population since it makes initialization and mutation processes very timeconsuming.

Table 1. Configuration set for the impulse noise removal based on hybrid genetic algorithm

\begin{tabular}{cc}
\hline Size of the population $\left(\mathrm{P}_{\mathrm{s}}\right)$ & 30 \\
\hline Mutation rate $\left(\mathrm{P}_{\mathrm{m}}\right)$ & 0.02 \\
Completion-criteria $\left(\right.$ iter $\left._{\max }\right)$ & when the algorithm reaches $=$ \\
number of iterations & 20 generations \\
$\beta$ & 1 \\
$\lambda$ & 0.08 \\
Selection criteria & Roulette Wheel selection \\
\hline
\end{tabular}

Table 1 shows the parameter settings for the proposed algorithm. We selected the values of these parameters by performing initial experiments taking into account the tradeoff between time and efficiency

\section{EXPERIMENTAL RESULTS}

In this section, we first presented six test images exposed. The first four of these images are among the most popular images. The second two of them are from TEST IMAGES [5, 28], as Figure 1 and Figure 2 shown, respectively.

Figure 3 and Figure 4 give the results of AMF, MDBUTMF, NAFSMF, and INRHGA for Girl face and Chair image with $80 \%$ and $90 \%$ densities by model 1 and model 2 of impulse noise, respectively. INRHGA preserved better the details of the image compared to other methods.

Moreover, Figure 5 and Figure 6 illustrates the results of INRHGA considering the input image Lenna and Billiard-Ball with noise densities $(20 \%, 40 \%, 60 \%, 80 \%$ and $90 \%)$ by model 1 and 2 of impulse noise, respectively

Afterwards, in Tables 2 and 3, we give results PSNR and SSIM of the methods of model 1, for Bridge, Couple, Girl face, and Lenna images ranging in noise densities from $10 \%$ to $90 \%$. Moreover, in Tables 4 and 5, we give the results PSNR and SSIM of the methods of model 2 for the same as test images. The results show that INRHGA performs better than the others at all noise densities in model 1 above $10 \%$ and all noise densities in model 2 above $30 \%$. 
Peak signal to noise ratio (PSNR) is defined as:

$$
P S N R=10 \cdot \log \left(\frac{255^{2}}{M S E}\right)
$$

where, MSE (Mean Square Error) is defined as:

$$
M S E=\frac{1}{M \times N} \sum_{i=0}^{M-1} \sum_{j=0}^{N-1}[O(i, j)-I(i, j)]^{2}
$$

where, $\mathrm{O}(\mathrm{i}, \mathrm{j})$ and $\mathrm{I}(\mathrm{i}, \mathrm{j})$ are the original image and the recovered image, respectively. Where $\mathrm{M}$ and $\mathrm{N}$ are the image dimensions.

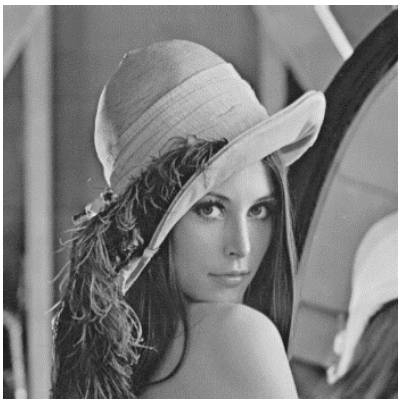

(a) Lenna

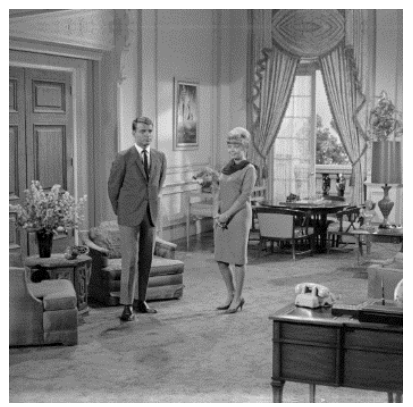

(b) Couple

Structural similarity index metric (SSIM), which can be mathematically formulated $[6,29]$, is defined as:

$$
\operatorname{SSIM}(x, y)=\frac{\left(2 \mu_{x} \mu_{y}+c_{1}\right)\left(2 \sigma_{x y}+c_{2}\right)}{\left(\mu_{x}^{2}+\mu_{y}^{2}+c_{1}\right)\left(\sigma_{x}^{2}+\sigma_{y}^{2}+c_{2}\right)}
$$

where, $\mu_{x}, \mu_{y}, \sigma_{x}^{2}, \sigma_{y}^{2}$, and $\sigma_{x y}$ are the mean intensities, standard deviations and covariance for images $\mathrm{x}$ and $\mathrm{y}$, respectively. $c_{1}=\left(k_{1} L\right)^{2}$ and $c_{2}=\left(k_{2} L\right)^{2}$ that $\mathrm{L}=255$ for 8 bit grayscale images and $k_{1}=0.01$ and $k_{2}=0.03$ are constant.

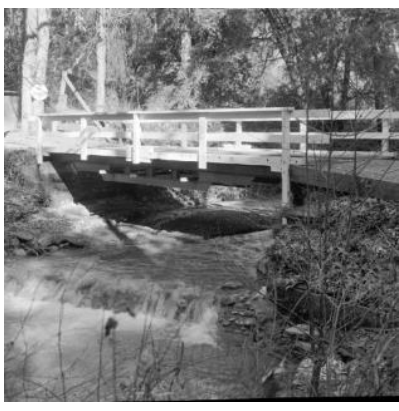

(c) Bridge

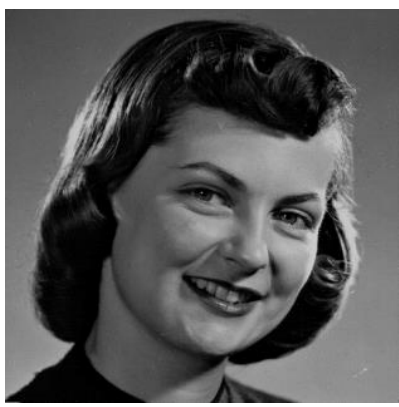

(d) Girlface

Figure 1. Classic test images

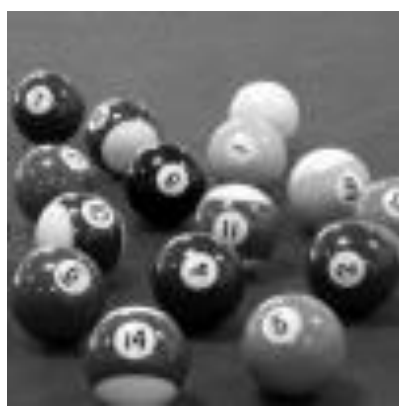

(a) Billiard-Ball

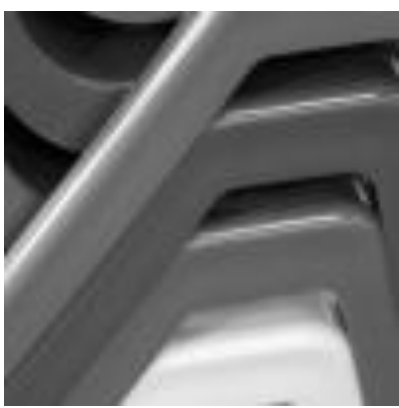

(b) Chair

Figure 2. TESTIMAGES Database

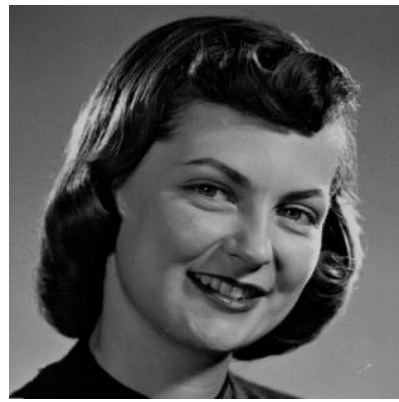

Originale image

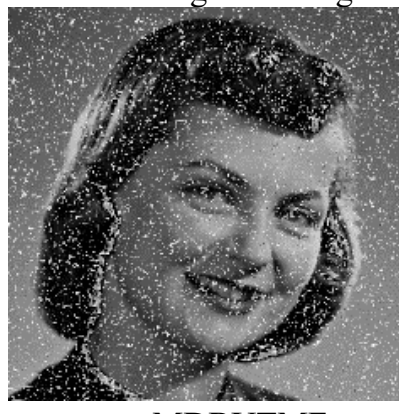

MDBUTMF

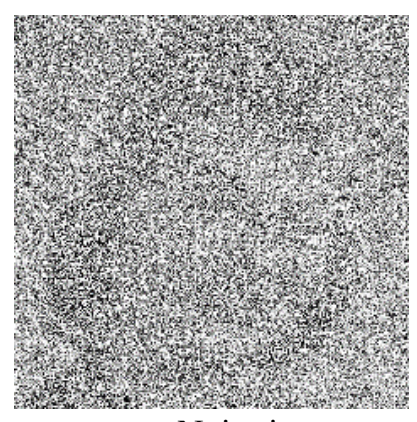

Noise image

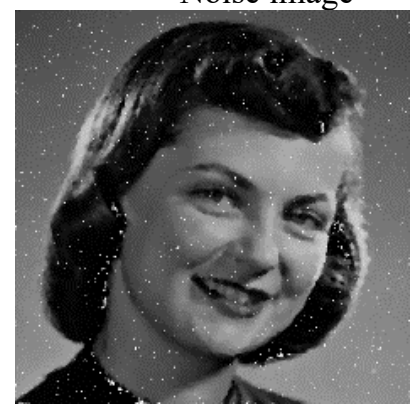

NAFSM

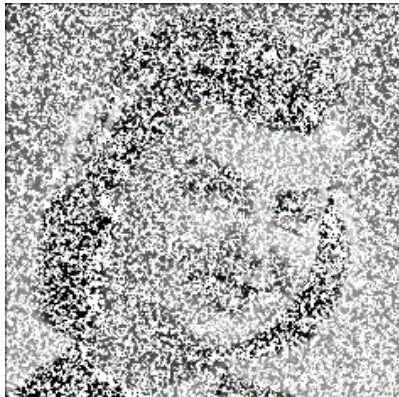

AMF

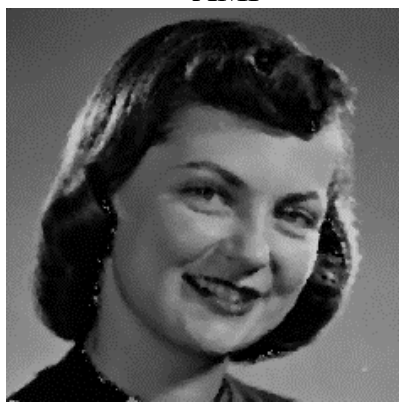

INRHGA

Figure 3. Restoration results of Girlface image perturbed by model 1 impulse noise with $80 \%$ densities 


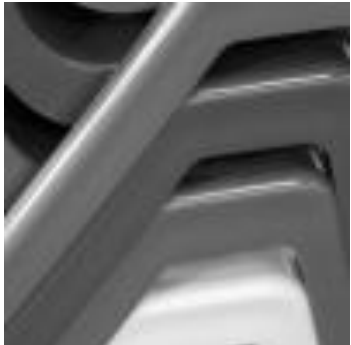

Originale image

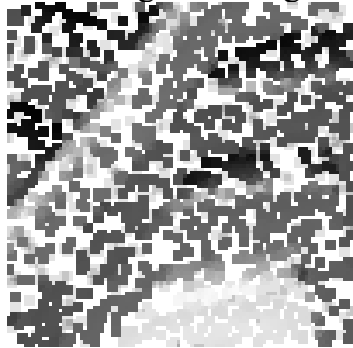

MDBUTMF

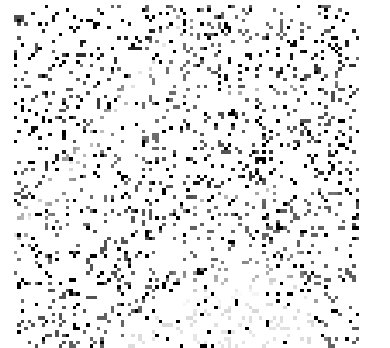

Noise image

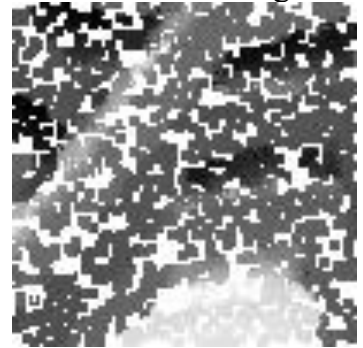

NAFSM
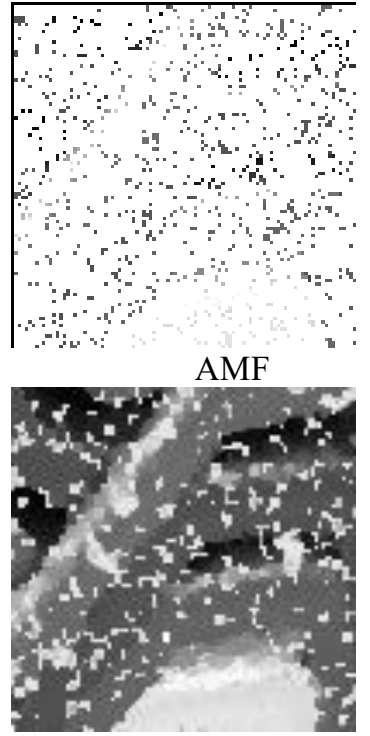

INRHGA

Figure 4. Restoration results of chair image perturbed by model 2 impulse noise with $90 \%$ densities

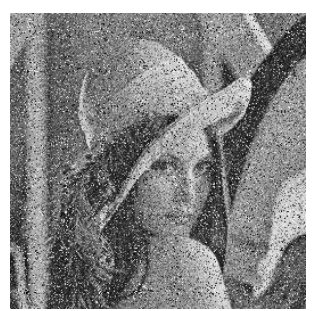

$20 \%$ noise density

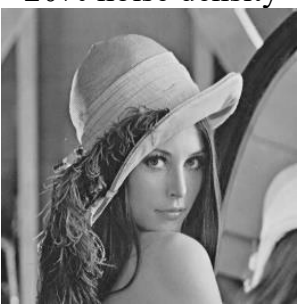

removed $20 \%$

PSNR-39.27,

SSIM-0.9786

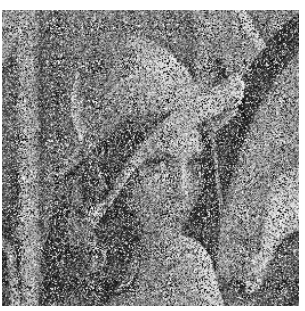

$40 \%$ noise density

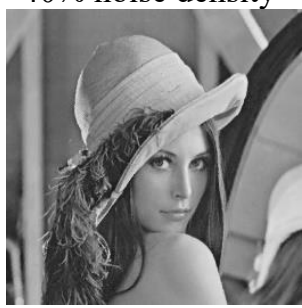

removed $40 \%$

PSNR-34.69,

SSIM-0.9466

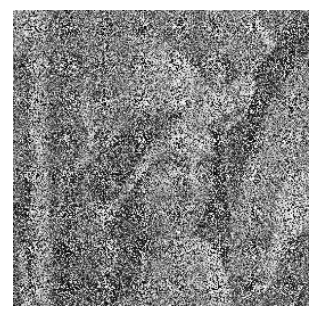

$60 \%$ noise density

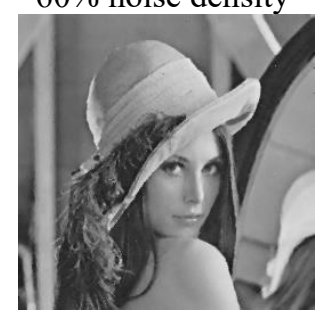

removed $60 \%$

PSNR-29.92,

SSIM-0.8818

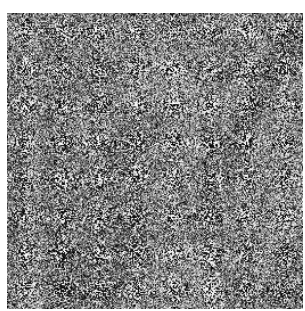

$80 \%$ noise density

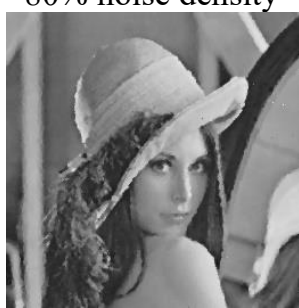

removed $80 \%$

PSNR-27.47,

SSIM-0.8125

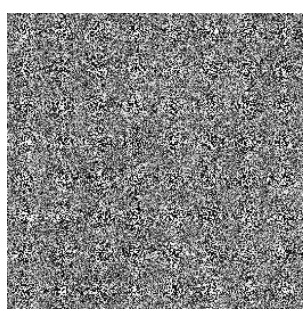

$90 \%$ noise density

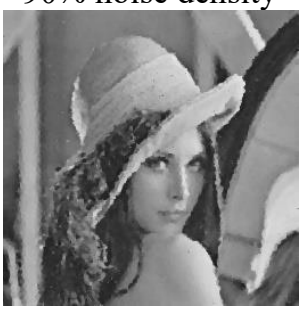

removed $90 \%$

PSNR-23.50,

SSIM-0.6823

Figure 5. Lenna perturbed by impulse noise of model 1, and Lenna images after INRHGA

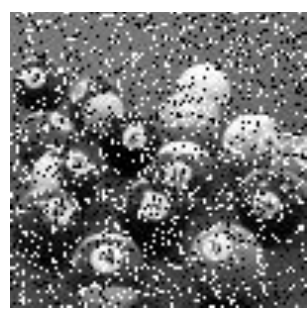

$20 \%$ noise density

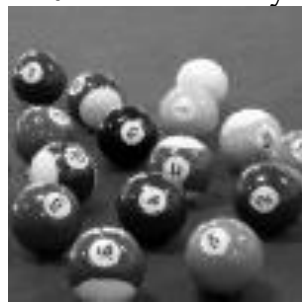

removed 20\%

PSNR-34.31,

SSIM-0.9771

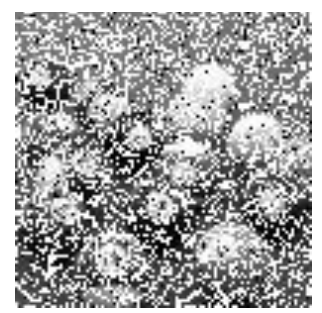

$40 \%$ noise density

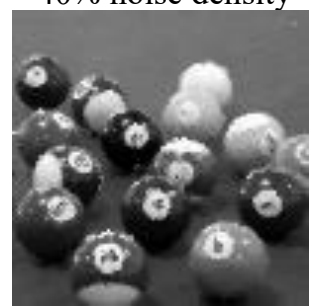

removed $40 \%$

PSNR-26.07,

SSIM-0.9375

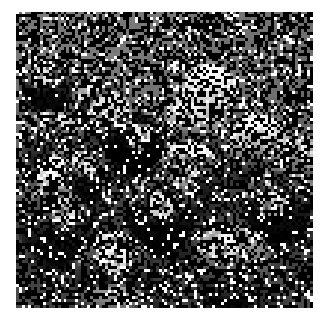

$60 \%$ noise density

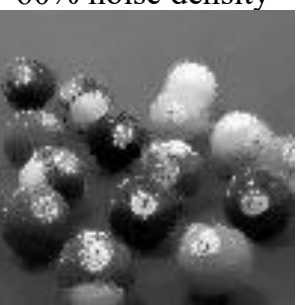

removed $60 \%$

PSNR-21.35,

SSIM-0.8131

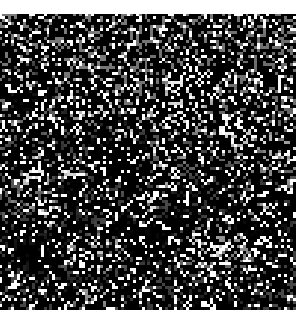

$80 \%$ noise density

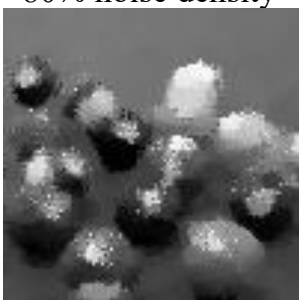

removed $80 \%$

PSNR-19.03,

SSIM-0.7002

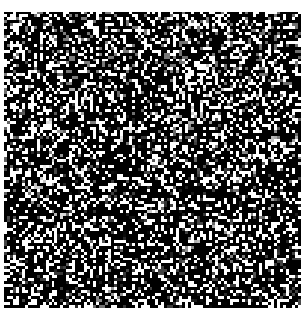

$90 \%$ noise density

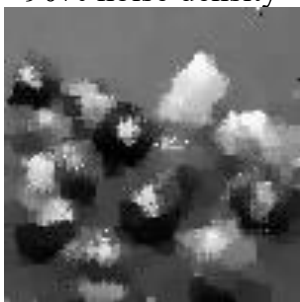

removed $90 \%$

PSNR-17.71,

SSIM-0.5985

Figure 6. Billiard-Ball perturbed by impulse noise of model 2, and Billiard-Ball images after INRHGA 
Table 2. PSNR results in the methods of model 1 for some images

\begin{tabular}{ccccccccccc}
\hline Image & Filter & $10 \%$ & $20 \%$ & $30 \%$ & $40 \%$ & $50 \%$ & $60 \%$ & $70 \%$ & $80 \%$ & $90 \%$ \\
\hline Bridge & AMF & 20,1999 & 20,1086 & 19,9944 & 19,849 & 19,373 & 17,8678 & 15,0618 & 11,577 & 8,1826 \\
& MDBUTMF & 34,1312 & 31,0621 & 28,6614 & 26,9952 & 25,454 & 23,5619 & 21,1819 & 18,1645 & 14,6439 \\
& NAFSM & 31,4271 & 28,6628 & 26,8445 & 25,5179 & 24,4377 & 23,4427 & 22,582 & 21,472 & 19,5182 \\
& INRHGA & 33,9909 & 30,8605 & 28,8919 & 27,0549 & 25,4826 & 23,6082 & 22,6709 & 21,7622 & 20,3202 \\
Couple & AMF & 22,4939 & 22,4284 & 22,332 & 22,1433 & 21,5342 & 19,5363 & 15,9518 & 12,0989 & 8,578 \\
& MDBUTMF & 38,5303 & 35,1063 & 32,6023 & 30,7843 & 27,2103 & 26,2491 & 23,5481 & 19,8943 & 16,0675 \\
& NAFSM & 34,3544 & 31,5208 & 29,5767 & 28,3064 & 27,1942 & 26,2143 & 25,2502 & 24,1182 & 21,5846 \\
& INRHGA & 38,5815 & 35,1237 & 32,6975 & 30,8103 & 28,9488 & 26,6344 & 25,3306 & 24,3105 & 22,873 \\
Girlface & AMF & 25,8914 & 25,8499 & 25,7482 & 25,4966 & 24,0687 & 20,5702 & 15,9459 & 11,7346 & 7,973 \\
& MDBUTMF & 38,8938 & 34,7307 & 32,9888 & 30,2575 & 27,9465 & 25,098 & 21,529 & 17,6643 & 13,7966 \\
& NAFSM & 37,7447 & 35,0151 & 33,3937 & 31,9485 & 30,985 & 29,7196 & 27,9988 & 26,031 & 21,8677 \\
& INRHGA & 38,9201 & 35,399 & 33,6443 & 32,2003 & 31,0969 & 30,1319 & 28,0003 & 27,4157 & 24,7181 \\
Lenna & AMF & 24,4338 & 24,3768 & 24,2976 & 24,0807 & 22,9636 & 20,3858 & 16,4434 & 12,1157 & 8,4666 \\
& MDBUTMF & 42,9759 & 39,0355 & 36,6427 & 34,6372 & 31,0664 & 28,7388 & 24,7712 & 20,1186 & 15,8785 \\
& NAFSM & 38,8213 & 35,6225 & 33,7468 & 32,323 & 31,0581 & 29,8547 & 28,639 & 27,0991 & 23,5366 \\
& INRHGA & 42,9204 & 39,2671 & 36,7048 & 34,6952 & 32,1341 & 29,9186 & 28,7633 & 27,4698 & 23,4983 \\
Mean & AMF & 23,2548 & 23,1910 & 23,0931 & 22,8924 & 21,9849 & 19,5900 & 15,8507 & 11,8816 & 08,3000 \\
& MDBUTMF & 38,6328 & 34,9837 & 32,7238 & 30,6686 & 27,9193 & 25,9120 & 22,7576 & 18,9604 & 15,0966 \\
& NAFSM & 35,5869 & 32,7053 & 30,8904 & 29,5240 & 28,4188 & 27,3079 & 26,1175 & 24,6801 & 21,6268 \\
& INRHGA & 38,6032 & 35,1626 & 32,9846 & 31,1902 & 29,4156 & 27,5733 & 26,1913 & 25,2396 & 22,8524 \\
\hline
\end{tabular}

Table 3. SSIM results in the methods of model 1 for some images

\begin{tabular}{ccccccccccc}
\hline Image & Filter & $10 \%$ & $20 \%$ & $30 \%$ & $40 \%$ & $50 \%$ & $60 \%$ & $70 \%$ & $80 \%$ & $90 \%$ \\
\hline Bridge & AMF & 0,4901 & 0,4823 & 0,4765 & 0,4698 & 0,4477 & 0,3857 & 0,2663 & 0,1242 & 0,0386 \\
& MDBUTMF & 0,9789 & 0,9535 & 0,9208 & 0,8839 & 0,8348 & 0,7194 & 0,6147 & 0,4207 & 0,2037 \\
& NAFSM & 0,9622 & 0,9225 & 0,8774 & 0,8311 & 0,778 & 0,7185 & 0,6483 & 0,5678 & 0,4337 \\
& INRHGA & 0,978 & 0,9528 & 0,9224 & 0,8857 & 0,8354 & 0,7536 & 0,6523 & 0,5731 & 0,4556 \\
Couple & AMF & 0,6086 & 0,6028 & 0,5974 & 0,5881 & 0,5614 & 0,4694 & 0,291 & 0,1146 & 0,0353 \\
& MDBUTMF & 0,9865 & 0,9682 & 0,9446 & 0,9164 & 0,8514 & 0,7889 & 0,6556 & 0,4283 & 0,2031 \\
& NAFSM & 0,9715 & 0,941 & 0,9065 & 0,8712 & 0,8318 & 0,7872 & 0,735 & 0,6642 & 0,5328 \\
& INRHGA & 0,9868 & 0,9684 & 0,9456 & 0,9173 & 0,8754 & 0,7978 & 0,7372 & 0,674 & 0,5785 \\
Girlface & AMF & 0,8117 & 0,8068 & 0,8015 & 0,7926 & 0,7425 & 0,6065 & 0,3393 & 0,1197 & 0,0307 \\
& MDUTMF & 0,9788 & 0,9643 & 0,9481 & 0,9295 & 0,8888 & 0,7925 & 0,5933 & 0,3292 & 0,1289 \\
& NAFSM & 0,9808 & 0,9636 & 0,9488 & 0,9305 & 0,9133 & 0,8919 & 0,8642 & 0,8258 & 0,7048 \\
& INRHGA & 0,9797 & 0,9651 & 0,9499 & 0,9308 & 0,9128 & 0,8925 & 0,8643 & 0,8398 & 0,7655 \\
Lenna & AMF & 0,7606 & 0,7544 & 0,7501 & 0,739 & 0,6941 & 0,5679 & 0,3325 & 0,116 & 0,0308 \\
& MDBUTMF & 0,9923 & 0,9805 & 0,9671 & 0,9486 & 0,9081 & 0,8312 & 0,6622 & 0,3982 & 0,17 \\
& NAFSM & 0,9859 & 0,9684 & 0,9504 & 0,9304 & 0,9078 & 0,8818 & 0,8511 & 0,8017 & 0,6819 \\
& INRHGA & 0,9922 & 0,9806 & 0,9671 & 0,9486 & 0,9141 & 0,8838 & 0,8546 & 0,8145 & 0,6843 \\
Mean & AMF & 0,6678 & 0,6616 & 0,6564 & 0,6474 & 0,6114 & 0,5074 & 0,3073 & 0,1186 & 0,0339 \\
& MDBUTMF & 0,9841 & 0,9666 & 0,9452 & 0,9196 & 0,8708 & 0,7830 & 0,6315 & 0,3941 & 0,1764 \\
& NAFSM & 0,9751 & 0,9489 & 0,9208 & 0,8908 & 0,8577 & 0,8199 & 0,7747 & 0,7149 & 0,5883 \\
& INRHGA & 0,9842 & 0,9667 & 0,9463 & 0,9206 & 0,8844 & 0,8319 & 0,7771 & 0,7254 & 0,6210 \\
\hline
\end{tabular}

Table 4. PSNR results in the methods of model 2 for some images

\begin{tabular}{ccccccccccc}
\hline Image & Filter & $10 \%$ & $20 \%$ & $30 \%$ & $40 \%$ & $50 \%$ & $60 \%$ & $70 \%$ & $80 \%$ & $90 \%$ \\
\hline Bridge & AMF & 20,2292 & 20,1175 & 19,7925 & 19,7336 & 15,5264 & 12,2532 & 8,3407 & 8,1846 & 8,2234 \\
& MDBUTMF & 34,2978 & 30,9631 & 28,4768 & 27,2498 & 24,7438 & 23,3324 & 19,1457 & 17,194 & 14,686 \\
& NAFSM & 31,6324 & 28,6236 & 27,1551 & 25,668 & 24,6961 & 23,3968 & 21,3106 & 20,4548 & 19,576 \\
& INRHGA & 34,2485 & 31,1783 & 29,1414 & 27,4299 & 25,6565 & 23,4906 & 22,6891 & 21,9699 & 20,2707 \\
Couple & AMF & 22,4913 & 22,3805 & 21,6792 & 21,4383 & 21,5016 & 9,7861 & 14,3058 & 6,4795 & 7,7111 \\
& MDBUTMF & 38,8286 & 34,9707 & 32,6247 & 30,6118 & 27,1889 & 24,2666 & 23,225 & 15,3942 & 15,3997 \\
& NAFSM & 34,6116 & 31,4578 & 29,5988 & 28,2863 & 27,2266 & 24,7258 & 25,229 & 18,2933 & 20,9087 \\
& INRHGA & 38,6923 & 35,0506 & 32,6346 & 30,8723 & 28,9528 & 26,2601 & 25,3163 & 24,2971 & 22,7802 \\
Girlface & AMF & 25,9081 & 25,7956 & 24,3552 & 21,4275 & 12,3631 & 8,3342 & 15,18 & 12,6008 & 8,9335 \\
& MDBUMF & 40,9269 & 39,2026 & 36,7941 & 27,8005 & 24,3342 & 20,9944 & 23,3093 & 19,1281 & 15,0042 \\
& NAFSM & 37,9844 & 35,763 & 33,9727 & 31,4295 & 28,8787 & 26,044 & 28,4429 & 26,3813 & 19,1511 \\
& INRHGA & 37,2819 & 38,2856 & 33,6955 & 31,9982 & 31,3146 & 29,8498 & 28,5719 & 28,1635 & 24,5959 \\
Lenna & AMF & 24,4611 & 24,3632 & 24,2775 & 23,6891 & 21,7659 & 17,15 & 16,1279 & 6,3904 & 5,7879 \\
& MDBUTMF & 42,9293 & 39,1671 & 36,761 & 34,501 & 30,942 & 28,4891 & 24,5797 & 15,0837 & 11,1496 \\
& NAFSM & 38,7396 & 35,5846 & 33,7161 & 32,3598 & 30,8756 & 29,6132 & 28,6649 & 17,3033 & 14,2669 \\
& INRHGA & 42,7286 & 39,1743 & 36,7786 & 34,6075 & 31,9927 & 29,9361 & 28,7636 & 27,4954 & 25,3531 \\
Mean & AMF & 23,2724 & 23,1642 & 22,5261 & 21,5721 & 17,7893 & 11,8809 & 13,4886 & 8,4138 & 7,6640 \\
& MDBUTMF & 39,2457 & 36,0759 & 33,6642 & 30,0408 & 26,8022 & 24,2706 & 22,5649 & 16,7000 & 14,0599 \\
& NAFSM & 35,7420 & 32,8573 & 31,1107 & 29,4359 & 27,9193 & 25,9450 & 25,9119 & 20,6082 & 18,4757 \\
& INRHGA & 38,2378 & 35,9222 & 33,0625 & 31,2270 & 29,4792 & 27,3842 & 26,3352 & 25,4815 & 23,2500 \\
\hline
\end{tabular}


Table 5. SSIM results in the methods of model 2 for four some images

\begin{tabular}{ccccccccccc}
\hline Image & Filter & $10 \%$ & $20 \%$ & $30 \%$ & $40 \%$ & $50 \%$ & $60 \%$ & $70 \%$ & $80 \%$ & $90 \%$ \\
\hline Bridge & AMF & 0,4917 & 0,4824 & 0,4711 & 0,4638 & 0,2959 & 0,1634 & 0,0847 & 0,0486 & 0,0426 \\
& MDBUTMF & 0,9785 & 0,9519 & 0,9218 & 0,885 & 0,8186 & 0,7191 & 0,5615 & 0,4037 & 0,2056 \\
& NAFSM & 0,9618 & 0,9207 & 0,8781 & 0,8303 & 0,7762 & 0,7041 & 0,6161 & 0,5025 & 0,4365 \\
& INRHGA & 0,979 & 0,9529 & 0,9212 & 0,8832 & 0,8306 & 0,7369 & 0,6513 & 0,5723 & 0,4534 \\
Couple & AMF & 0,6087 & 0,6044 & 0,5766 & 0,5623 & 0,5584 & 0,0729 & 0,2346 & 0,0357 & 0,0288 \\
& MDBUTMF & 0,9869 & 0,9675 & 0,9447 & 0,9149 & 0,8307 & 0,7413 & 0,6424 & 0,2798 & 0,1874 \\
& NAFSM & 0,9726 & 0,9407 & 0,9066 & 0,8711 & 0,8322 & 0,7366 & 0,731 & 0,3968 & 0,4899 \\
& INRHGA & 0,9871 & 0,968 & 0,9451 & 0,9182 & 0,875 & 0,7876 & 0,737 & 0,6733 & 0,5769 \\
Girlface & AMF & 0,8127 & 0,8078 & 0,746 & 0,6806 & 0,2095 & 0,055 & 0,2848 & 0,1653 & 0,0887 \\
& MDBUTMF & 0,9843 & 0,9805 & 0,948 & 0,9232 & 0,8676 & 0,716 & 0,6253 & 0,3541 & 0,1481 \\
& NAFSM & 0,9812 & 0,9645 & 0,9454 & 0,9295 & 0,8991 & 0,8341 & 0,854 & 0,8068 & 0,405 \\
& INRHGA & 0,9798 & 0,9738 & 0,9667 & 0,9314 & 0,9115 & 0,8935 & 0,8665 & 0,8272 & 0,767 \\
Lenna & AMF & 0,7624 & 0,7558 & 0,7488 & 0,729 & 0,6417 & 0,4009 & 0,3239 & 0,0419 & 0,0662 \\
& MDBUTMF & 0,9923 & 0,9804 & 0,967 & 0,9476 & 0,908 & 0,8269 & 0,6542 & 0,2452 & 0,1013 \\
& NAFSM & 0,9859 & 0,9682 & 0,9502 & 0,9304 & 0,9066 & 0,8788 & 0,8497 & 0,352 & 0,1874 \\
& INRHGA & 0,9919 & 0,9804 & 0,9671 & 0,9484 & 0,9136 & 0,8837 & 0,8535 & 0,816 & 0,7443 \\
Mean & AMF & 0,6689 & 0,6626 & 0,6356 & 0,6089 & 0,4264 & 0,1731 & 0,2320 & 0,0729 & 0,0566 \\
& MDBUTMF & 0,9855 & 0,9701 & 0,9454 & 0,9177 & 0,8562 & 0,7508 & 0,6209 & 0,3207 & 0,1606 \\
& NAFSM & 0,9754 & 0,9485 & 0,9201 & 0,8903 & 0,8535 & 0,7884 & 0,7627 & 0,5145 & 0,3797 \\
& INRHGA & 0,9845 & 0,9688 & 0,9500 & 0,9203 & 0,8827 & 0,8254 & 0,7771 & 0,7222 & 0,6354 \\
\hline
\end{tabular}

\section{CONCLUSIONS}

In this paper, we have proposed a new impulse noise removal by applying a hybrid genetic algorithm (INRHGA), we address the reduction of impulse noise in images as an optimization problem, which gives better performance in comparison with known noise removal methods in terms of PSNR and SSIM. The performance of the algorithm has been tested an all noise densities on grayscale images. The proposed method is effective for impulse noise removal. An important advantage of INRHGA is impulse noise removal in noisy image without the original image, so it works without a clue about how far we are from the original image.

Finally, our scope of work did not focus on the arithmetic cost of the algorithm, but on the quality of the recovered images. We intend to verify the computational cost and reduce the current implementation time of the proposed algorithm as future work.

\section{REFERENCES}

[1] Fareed, S.B.S., Khader, S.S. (2018). Fast adaptive and selective mean filter for the removal of high-density salt and pepper noise. IET Image Processing, 12(8): 13781387.

[2] Chen, J., Zhan, Y., Cao, H., Wu, X. (2018). Adaptive probability filter for removing salt and pepper noises. IET Image Processing, 12(6): 863-871.

[3] Mafi, M., Tabarestani, S., Cabrerizo, M., Barreto, A., Adjouadi, M. (2018). Denoising of ultrasound images affected by combined speckle and Gaussian noise. IET Image Processing, 12(12): 2346-2351.

[4] Ahmed, F., Das, S. (2013). Removal of high-density saltand-pepper noise in images with an iterative adaptive fuzzy filter using alpha-trimmed mean. IEEE Transactions on Fuzzy Systems, 22(5): 1352-1358. https://doi.org/10.1109/TFUZZ.2013.2286634

[5] Erkan, U., Gökrem, L., Enginoğlu, S. (2019). Adaptive right median filter for salt-and-pepper noise removal. International Journal of Engineering Research and
Development, $\quad 11(2)$ :

https://doi.org/10.29137/umagd.495904

$542-550$

[6] Thanh, D.N.H., Engínoğlu, S. (2019). An iterative mean filter for image denoising. IEEE Access, 7: 167847167859.

https://doi.org/10.1109/ACCESS.2019.2953924

[7] Erkan, U., Gökrem, L., Enginoğlu, S. (2018). Different applied median filter in salt and pepper noise. Computers \& Electrical Engineering, 70: 789-798. https://doi.org/10.1016/j.compeleceng.2018.01.019

[8] Abdurrazzaq, A., Mohd, I., Junoh, A.K., Yahya, Z. (2018). Modified tropical algebra based median filter for removing salt and pepper noise in digital image. IET Image Processing, 13(14): 2790-2795. https://doi.org/10.1049/iet-ipr.2018.6201

[9] Chen, J., Zhan, Y., Cao, H., Xiong, G. (2019). Iterative grouping median filter for removal of fixed value impulse noise. IET Image Processing, 13(6): 946-953.

[10] Naimi, H., Adamou-Mitiche, A.B.H., Mitiche, L. (2015). Medical image denoising using dual tree complex thresholding wavelet transform and Wiener filter. Journal of King Saud University-Computer and Information Sciences, 27(1): 40-45. https://doi.org/10.1016/j.jksuci.2014.03.015

[11] Lone, A.H., Siddiqui, A.N. (2018). Noise models in digital image processing. Global Sci-Tech, 10(2): 63-66. https://doi.org/10.5958/2455-7110.2018.00010.1

[12] Bovik, A.C. (2010). Handbook of Image and Video Processing. Academic Press. 2005, p. vii.

[13] Hwang, H., Haddad, R.A. (1995). Adaptive median filters: New algorithms and results. IEEE Transactions on Image Processing, 4(4): 499-502. https://doi.org/10.1109/83.370679

[14] Pattnaik, A., Agarwal, S., Chand, S. (2012). A new and efficient method for removal of high density salt and pepper noise through cascade decision based filtering algorithm. Procedia Technology, 6: 108-117. https://doi.org/10.1016/j.protcy.2012.10.014

[15] Toh, K.K.V., Isa, N.A.M. (2009). Noise adaptive fuzzy switching median filter for salt-and-pepper noise reduction. IEEE Signal Processing Letters, 17(3): 281- 
284. https://doi.org/10.1109/LSP.2009.2038769

[16] Toledo, C.F., de Oliveira, L., da Silva, R.D., Pedrini, H. (2013). Image denoising based on genetic algorithm. In 2013 IEEE Congress on Evolutionary Computation, pp. 1294-1301. https://doi.org/10.1109/CEC.2013.6557714

[17] de Paiva, J.L., Toledo, C.F., Pedrini, H. (2015). A hybrid genetic algorithm for image denoising. In 2015 IEEE Congress on Evolutionary Computation (CEC), pp. 2444-2451. https://doi.org/10.1109/CEC.2015.7257188

[18] Sakthidasan, K., Nagappan, N.V. (2016). Noise free image restoration using hybrid filter with adaptive genetic algorithm. Computers \& Electrical Engineering, 54:

382-392.

https://doi.org/10.1016/j.compeleceng.2015.12.011

[19] Fajardo-Delgado, D., Sánchez, M.G., Molinar-Solis, J.E., Fernandez-Zepeda, J.A., Vidal, V., Verdiú, G. (2016). A hybrid genetic algorithm for color image denoising. In 2016 IEEE Congress on Evolutionary Computation (CEC), $\quad$ pp. 3879-3886. https://doi.org/10.1109/CEC.2016.7744281

[20] de Paiva, J.L., Toledo, C.F., Pedrini, H. (2016). An approach based on hybrid genetic algorithm applied to image denoising problem. Applied Soft Computing, 46: 778-791. https://doi.org/10.1016/j.asoc.2015.09.013

[21] Sánchez, M.G., Fajardo-Delgado, D., Vidal, V., Verdú, G. (2020). A hybrid genetic algorithm to reduce the radiation dose in CR images. Radiation Physics and Chemistry, $\quad 167$ : 108275 https://doi.org/10.1016/j.radphyschem.2019.04.025

[22] Alaoui, N., Adamou-Mitiche, A.B.H., Mitiche, L. (2020). Effective hybrid genetic algorithm for removing salt and pepper noise. IET Image Processing, 14(2): 289-296.

[23] Saraiva, A.A., de Oliveira, M.S., de Moura Oliveira, P.
B., Solteiro Pires, E.J., Fonseca Ferreira, N.M., Valente, A. (2019). Genetic algorithm applied to remove noise in DICOM images. Journal of Information and Optimization Sciences, 40(7): 1543-1558. https://doi.org/10.1080/02522667.2019.1597999

[24] Verma, D., Vishwakarma, V.P., Dalal, S. (2020). A hybrid self-constrained genetic algorithm (HSGA) for digital image Denoising based on PSNR improvement. In Advances in Bioinformatics, Multimedia, and Electronics Circuits and Signals, 1064: 135-153. Springer, Singapore. https://doi.org/10.1007/978-98115-0339-9 12

[25] Shen, C., Wang, D., Tang, S., Cao, H., Liu, J. (2017). Hybrid image noise reduction algorithm based on genetic ant colony and PCNN. The Visual Computer, 33(11): 1373-1384. https://doi.org/10.1007/s00371-016-1325-x

[26] Zosso, D., Bustin, A. (2014). A primal-dual projected gradient algorithm for efficient Beltrami regularization. Computer Vision and Image Understanding, 14-52.

[27] Chan, T.F., Esedoglu, S. (2005). Aspects of total variation regularized L 1 function approximation. SIAM Journal on Applied Mathematics, 65(5): 1817-1837. https://doi.org/10.1137/040604297

[28] Asuni, N., Giachetti, A. (2014, September). TESTIMAGES: A Large-scale Archive for Testing Visual Devices and Basic Image Processing Algorithms. In STAG, 63-70.

[29] Wang, Z., Bovik, A.C., Sheikh, H.R., Simoncelli, E.P. (2004). Image quality assessment: From error visibility to structural similarity. IEEE Transactions on Image Processing, 13(4): 600-612. https://doi.org/10.1109/TIP.2003.819861 